\title{
Erratum to: Inaugural Conference on Incorporating Patient- Reported Outcomes and Patient Preference Information into Clinical Research, Clinical Care, and Risk-Benefit Assessments for Neurodegenerative Diseases
}

\section{Jennifer L. Purks ${ }^{1}$ Erin E. Wilhelm ${ }^{2}$ - Ira Shoulson ${ }^{2} \cdot$ John Creveling $^{3}$ • E. Ray Dorsey ${ }^{4}$ Telba Irony ${ }^{5}$ Tara M. LoCastro ${ }^{6} \cdot$ Fernando Pagan $^{1,7}$. Bernard Ravina $^{8} \cdot$ Tanya Simuni $^{9} \cdot$ MaryAnne Sterling $^{10} \cdot$ Pierre Tariot $^{11}$. Karen E. Anderson ${ }^{1}$}

Published online: 1 August 2017

(c) Springer International Publishing AG 2017

\section{Erratum to: Patient}

\section{DOI 10.1007/s40271-017-0257-5}

In the original publication, the affiliations 7 to 11 were incorrectly published and they have been corrected in this erratum.

Affiliations 7 to 11 which were previously stated as

${ }^{7}$ Voyager Therapeutics Inc., 75 Sidney Street, Cambridge, MA 02139, USA

${ }^{8}$ Department of Neurology, Northwestern University Feinberg School of Medicine, 710 North Lake Shore Drive, 1126, Chicago, IL 60611, USA
${ }^{9}$ Sterling Health IT, 42950 Tara Court, Ashburn, VA 20147, USA

${ }^{10}$ Banner Alzheimer's Institute, Banner Health, Phoenix, AZ 85006, USA

${ }^{11} 3800$ Reservoir Road, 7PHC, Washington, DC 20007, USA

Should read

${ }^{7}$ Department of Neurology, Georgetown University Medical Center, 3800 Reservoir Road, 7PHC, Washington, DC 20007, USA

The online version of the original article can be found under doi:10.1007/s40271-017-0257-5.

Jennifer L. Purks

jlp92@georgetown.edu

1 Huntington Disease Care, Education and Research Center, Georgetown University, 2115 Wisconsin Ave., NW, Suite 603, Washington, DC 20007, USA

2 Program for Regulatory Science and Medicine, Georgetown University, 2115 Wisconsin Ave., NW, Suite 603, Washington, DC 20007, USA

3 Philadelphia, PA 19146, USA

4 Center for Human Experimental Therapeutics, University of Rochester Medical Center, 265 Crittenden Blvd, Mail Stop CU 420694, Rochester, NY 14642, USA

5 Center for Biologics Evaluation and Research, Office of Biostatistics and Epidemiology, U.S. Food and Drug Administration, 10903 New Hampshire Ave, Silver Spring, MD 20993, USA
6 Galica Healthcare, 373 Bay Village Drive, Rochester, NY 14609, USA

7 Department of Neurology, Georgetown University Medical Center, 3800 Reservoir Road, 7PHC, Washington, DC 20007, USA

8 Voyager Therapeutics Inc., 75 Sidney Street, Cambridge, MA 02139, USA

9 Department of Neurology, Northwestern University Feinberg School of Medicine, 710 North Lake Shore Drive, 1126, Chicago, IL 60611, USA

10 Sterling Health IT, 42950 Tara Court, Ashburn, VA 20147, USA

11 Banner Alzheimer's Institute, Banner Health, Phoenix, AZ 85006, USA 
${ }^{8}$ Voyager Therapeutics Inc., 75 Sidney Street, Cambridge, MA 02139, USA

${ }^{9}$ Department of Neurology, Northwestern University Feinberg School of Medicine, 710 North Lake Shore Drive, 1126, Chicago, IL 60611, USA
${ }^{10}$ Sterling Health IT, 42950 Tara Court, Ashburn, VA 20147, USA

${ }^{11}$ Banner Alzheimer's Institute, Banner Health, Phoenix, AZ 85006, USA 